\title{
Large Deviations from Classical Paths. Hamiltonian Flows as Classical Limits of Quantum Flows
}

\author{
Ph. Blanchard ${ }^{1}$ and M. Sirugue ${ }^{2 \star}$ \\ 1 Theoretische Physik, Universität Bielefeld, D-4800 Bielefeld 1, Federal Republic of Germany \\ 2 Zentrum für interdisziplinäre Forschung, Universität Bielefeld, D-4800 Bielefeld 1, Federal Republic of \\ Germany
}

\begin{abstract}
We prove that in the limit $\hbar \rightarrow 0$, the probability for the paths of the stochastic jump process associated to the quantum time evolution to be in a tublet around the classical trajectory is of order $1-\exp \{-A / \hbar\}$. We give some applications of this result to the study of the classical limit of Wigner functions.
\end{abstract}

\section{Introduction}

In a previous paper [1] it was shown that the real time evolution of typical matrix elements of a relativistic quantum field theory with trigonometric interaction can be described in any space time dimension by a stochastic flow on the function space of initial conditions. More precisely, there exists a generalized stochastic process $(\Phi(x, t), \Pi(x, t))$ with value in the space of initial conditions and a functional $S$ of this process such that the expectation value at time $t$ in the ground state of the exponential of the field operator is given by

$$
\begin{aligned}
& (\Omega \\
& \left.\quad \exp \left\{i\left(\Phi_{t} f\right)-i\left(\Pi_{t} q\right)\right\} \Omega\right) \\
& \quad=e^{c t} \mathbb{E}\left[\exp \left\{\frac{i}{h} S_{t}\right\}\left(\Omega \mid \exp \left(i\left(\Phi \Phi_{t}\right)-i\left(\Pi \Pi_{t}\right)\right) \Omega\right) \Gamma_{t}=0\right],
\end{aligned}
$$

$c$ being a constant. This expression turned out to be very convenient to prove the existence of limits when the cutoffs required to define the interaction are removed.

In a second paper [2] we concentrated on the case of quantum mechanics, viz on the case of systems with a finite number of degrees of freedom. We have shown that it was more natural and useful to write the previous expression using a process in a

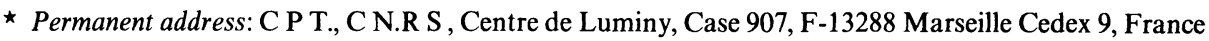


space where one auxiliary dimension has been added to the phase space. Thus defining a stochastic flow in $\mathbb{R}^{2 n+1}$, where $\mathbb{R}^{2 n}$ was the phase space of the classical system. In this way the original Feynman representation of the time evolution described by an integral over trajectories is made rigorous. This representation also has an appealing feature which already appeared in the original work of Feynman. Namely, it is intuitive that as $\hbar$ (the Planck's constant) becomes smaller and smaller, the probability on the phase space trajectories pinches on a tublet around the classical trajectory. This heuristic idea can be given a rigorous meaning as we shall show in this paper.

The natural mathematical framework for such an investigation is the theory of large deviations for stochastic processes. This theory has been developed quite extensively in recent years (see e.g. [3-7] and the references therein). It has been already used for physical applications in connection with the study of the classical limit of quantum mechanics ([8-11]) formulated in terms of diffusion processes. In this paper we shall use another point of view, viz the possibility of formulating the quantum time evolution for typical quantum mechanical matrix elements in terms of pure jump processes $([1,2])$. Consequently we shall use the theory of large deviations for this type of processes (see [4-6]).

This paper is organized as follows: In Sect. 2, besides the known results about the Wigner functions that we have introduced for the sake of completeness, we discuss an "interaction-like" picture in our framework. This is an essential technical tool for our later results. Furthermore, to give a probabilistic interpretation of the Schrödinger equation we introduce an extra dimension to the phase space $\mathbb{R}^{2 n}$ obtaining in this way a stochastic flow on $\mathbb{R}^{2 n+1}$. This is a natural technique which has been used already in different contexts (see e.g. $[12,13]$ ) as well as in [2]. Note that our representation looks a bit different from the one obtained in [2]. However, it is more adapted to the study of large deviations.

In Sect. 3 we give the most important result of this paper. The probability for the trajectories of the process to be inside a tublet around the classical trajectory is one up to order $\exp \{-A / \hbar\}$. In fact, as $\hbar$ goes to zero the typical trajectory has no component in the extra dimension. Consequently for the bounded continuous functions on $\mathbb{R}^{2 n+1}$, the flow tends to the classical one.

These results do not allow us to discuss directly the classical limit for the time evolution of Wigner functions. Indeed, the expectation value which defines the time evolution of the Wigner functions contains an oscillatory term depending on $\hbar$. However, it can be controlled completely at least for sufficiently smooth Wigner functions. This is done in Sect. 4. Moreover, for the sake of completeness we discuss a version of Ehrenfest's theorem and get in this way a connection with an earlier work by K. Hepp [14].

\section{Interaction Picture}

Here we consider a dynamical system whose classical phase space is $\mathbb{R}^{2 n}$. The canonical symplectic form $\sigma$ on $\mathbb{R}^{2 n}$ is:

$$
\sigma\left(a, a^{\prime}\right)=\sum_{i=1}^{n}\left\{q_{i}^{\prime} p_{i}-q_{i} p_{i}^{\prime}\right\}
$$


where $a=\left(q_{i}, p_{i}\right)_{i=1, \ldots n}$ (respectively $\left.a^{\prime}=\left(q_{i}^{\prime}, p_{i}^{\prime}\right)\right)$ in a given basis of $\mathbb{R}^{2 n}$.

The usual Weyl quantization procedure [16] associates with every function $f$ in $L^{1}\left(\mathbb{R}^{2 n}, d x\right)$ the operator $Q(f)$ such that

$$
Q(f)=(2 \pi)^{-n} \int_{\mathbb{R}^{2 n}} \tilde{f}\left(a^{\prime}\right) W_{a^{\prime}} d a^{\prime}
$$

where $f$ is defined as

$$
f(a)=(2 \pi)^{-n} \int_{\mathbb{R}^{2 n}} f\left(a^{\prime}\right) e^{i \sigma\left(a, a^{\prime}\right)} d a^{\prime}
$$

and $a \in \mathbb{R}^{2 n} \rightarrow W_{a}$ is a continuous unitary projective representation of the additive group of $\mathbb{R}^{2 n}$ :

$$
W_{a} W_{a^{\prime}}=\exp \left\{\frac{i \hbar}{2} \sigma\left(a, a^{\prime}\right)\right\} W_{a+a^{\prime}},
$$

where $\hbar$ is the Planck's constant $h$ divided by $2 \pi$.

It is well known that up to quasi-equivalence there exists only one unitary projective representation of the additive group of $\mathbb{R}^{2 n}$ admitting $\hbar \sigma$ as multiplier. This implies that any quantum state $\langle Q(f)\rangle$ is of the form

$$
\langle Q(f)\rangle=(\hbar \pi)^{-n} \int_{\mathbb{R}^{2 n}} d a f(a) \operatorname{tr}\left\{R W_{2 a / h}^{*} \Pi\right\},
$$

where $W_{a}$ is the usual representation of the canonical commutation relations in the Weyl form (2.4) on the Hilbert space $L^{2}\left(\mathbb{R}^{n}, d x\right), R$ a density matrix, i.e. a positive trace-class operator of trace one on $L^{2}\left(\mathbb{R}^{n}, d x\right)$ and $\Pi$ the parity operator

$$
(\Pi \psi)(x)=\psi(-x) \quad \psi \in L^{2}\left(\mathbb{R}^{n}, d x\right) .
$$

In particular, the Wigner function [15]

$$
W_{h}(a)=(\hbar \pi)^{-n} \operatorname{tr}\left(R W_{2 a / \hbar}^{*} \Pi\right)
$$

contains the whole physical information on the system in the state $R$. It depends explicitly on $\hbar$. The set of Wigner functions can be characterized completely as the set of Fourier transforms of continuous functions $\tilde{W}$ such that

1. $\tilde{W}(0)=1$

2. $\sum_{i, j=1}^{N} \lambda_{i} \bar{\lambda}_{j} \exp \left\{-\frac{i \hbar}{2} \sigma\left(a_{i}, a_{j}\right)\right\} \tilde{W}\left(a_{i}-a_{j}\right) \geqq 0$

for any choice $\left\{\lambda_{i}, a_{i}\right\}_{i=1, \ldots, N}, \lambda_{i} \in \mathbb{C}, a_{i} \in \mathbb{R}^{2 n}$. It will be useful in what follows to notice the obvious:

Lemma 2.1. Let $W_{h}$ be a Wigner function. Then $a \in \mathbb{R}^{2 n} \rightarrow W_{h}(a)$ is a continuous mapping of $\mathbb{R}^{2 n}$ in $\mathbb{R}$ such that $\left|W_{h}(a)\right| \leqq 1$. Furthermore, the set of Wigner functions is convex.

We shall be considering quantum dynamics which are given by the continuous group of unitaries

$$
t \in \mathbb{R} \rightarrow \exp \left\{\frac{i t}{\hbar} H\right\}
$$


where the Hamiltonian operator $H$ is of the form

$$
H=\frac{1}{2} \sum_{i=1}^{N}\left\{\frac{P_{i}^{2}}{m_{i}}+m_{i} \omega_{i}^{2} Q_{i}^{2}\right\}+V,
$$

$P_{i}$ and $Q_{i}$ being the usual momentum and position operators whereas

$$
V=\int_{\mathbb{R}^{2 n}} d \mu(a) W_{a},
$$

$\mu$ being a bounded measure of bounded variation on $\mathbb{R}^{2 n}$. By a measure of bounded variation we will mean one of the form $\mu=e^{i \varphi()}|\mu|$, where $\varphi$ is a real valued function, and where $|\mu|$ is a finite positive measure. Furthermore, we shall require that $V$, and hence $H$, is a self-adjoint operator. This implies that $|\mu|$ is a symmetric measure and $\varphi$ an antisymmetric function.

The potential $V$ is the quantized operator corresponding to the classical potential in phase space given by

$$
V^{c}(a)=\int_{\mathbb{R}^{2 n}} d|\mu|\left(a^{\prime}\right) \cos \left(\sigma\left(a, a^{\prime}\right)+\varphi\left(a^{\prime}\right)\right)
$$

and the Hamilton operator $H$ in (2.8) is the quantized operator corresponding to the following classical Hamilton function,

$$
H(q, p)=\sum_{i=1}^{n}\left\{\frac{p_{i}^{2}}{2 m_{i}}+\frac{m_{i} \omega_{i}^{2}}{2} q_{i}^{2}\right\}+V^{c}(q, p),(q, p) \in \mathbb{R}^{2 n} .
$$

On the other hand, let $W_{h}$ be a Wigner function; then it can be represented as in (2.7), and the mapping

$$
\begin{aligned}
t \in \mathbb{R} & \rightarrow W_{h t}^{H}(a), \\
W_{h t}(a) & =(\hbar \pi)^{-n} \operatorname{tr}\left(R \exp \left\{\frac{i t}{\hbar} H\right\} W_{2 a / \hbar}^{*} \Pi \exp \left\{-\frac{i t}{\hbar} H\right\}\right)
\end{aligned}
$$

defines a group of affine continuous mappings of the set of Wigner functions.

Let us first observe that if $V \equiv 0$, then

$$
W_{h t}^{0}(a)=W_{h}\left(a_{t}\right) \quad a \in \mathbb{R}^{2 n},
$$

where

$$
(q, p)_{t}=\left(q_{i} \cos \left(\omega_{i} t\right)-\frac{1}{m_{i} \omega_{i}} \sin \left(\omega_{i} t\right) p_{i}, m_{i} \omega_{i} q_{i} \sin \left(\omega_{i} t\right)+\cos \left(\omega_{i} t\right) p_{i}\right)
$$

independently of $\hbar$. A similar property holds for any quantum Hamilton operator which is at most quadratic in the $P$ 's and $Q$ 's.

For what follows it is convenient to consider an "interaction-like picture" which takes into account this free motion.

Let $H_{0}$ be the Hamiltonian (2.8) with $V \equiv 0$. Then

$$
\begin{aligned}
t \in \mathbb{R} & \rightarrow W_{h t}^{I}(a), \\
W_{h t}^{I}(a) & =(\hbar \pi)^{-n} \operatorname{tr}\left(R e^{-i t H / \hbar} e^{+i t H_{0} / \hbar} W_{2 a / \hbar}^{*} \Pi e^{-i t H_{0} / \hbar} e^{i t H / \hbar}\right.
\end{aligned}
$$


defines a family of affine continuous mappings of the Wigner functions.

For convenience we introduce for each $T \in \mathbb{R}$ and $t \leqq T$ the mapping $t \rightarrow W_{h, t-T}^{I}$, which satisfies $\lim _{t \rightarrow T} W_{h, t-T}^{I}(a)=W_{h}(a)$.

The time evolution is governed by an integro-differential equation which is a Schrödinger equation in the interaction picture for Wigner functions. We call it Schrödinger equation since it defines the time evolution of the states and not of the observables. More precisely, we have the

Proposition 2.2 (see $[1,2]) . W_{h, t-T}^{I}(a), \forall a \in \mathbb{R}^{2 n}$ satisfies the following integrodifferential equation

$$
\frac{\partial}{\partial t} W_{h, t-T}^{I}(a)-\frac{2}{\hbar} \int_{\mathbb{R}^{2 n}} d|\mu|\left(a^{\prime}\right) \sin \left(\sigma\left(a_{T-t}^{\prime}, a\right)+\varphi\left(a^{\prime}\right)\right) W_{h, t-T}^{I}\left(a+\frac{\hbar}{2} a_{T-t}^{\prime}\right)=0
$$

with the final condition $\lim _{t \rightarrow T} W_{h, t-T}^{I}(a)=W_{h}(a)$.

To interpret this equation probabilistically as a backward Kolmogorov equation for a Markov stochastic process, let us introduce an auxiliary dimension and define the function $\Xi:]-\infty, T] \times \mathbb{R}^{2 n+1} \rightarrow \mathbb{C}$,

$$
\Xi(t, q, p, s)=\exp \left\{4\left(\frac{t-T}{\hbar}\right)|\mu|+\frac{i}{h} s\right\} W_{h t}^{I}(q, p),
$$

with $|\mu|$ defined as

$$
|\mu|=\int d|\mu|\left(a^{\prime}\right)
$$

$\Xi$ is a bounded continuous function such that $\lim _{t \uparrow T} \Xi(t, q, p, s=0)=W_{h t}(q, p)$. The time evolution of $\Xi$ is described in the following

Proposition 2.3. Let $v_{\text {tqps }}$ be the bounded positive measure on $\mathbb{R}^{2 n+1}$ defined as:

$$
\begin{aligned}
d v_{t q p s}\left(q^{\prime}, p^{\prime}, s^{\prime}\right)= & \left(\frac{2}{\hbar}\right)^{2 n+1} d|\mu|\left(\frac{2}{\hbar} a_{t-T}^{\prime}\right)\left(1-\sin \left(\frac{2}{\hbar} \sigma\left(a^{\prime}, a\right)+\varphi\left(\frac{2}{\hbar} a_{t-T}^{\prime}\right)\right)\right) \beta_{\hbar}\left(s^{\prime}\right) \\
& +\left(\frac{2}{\hbar}\right)^{2 n+1} d|\mu|\left(\frac{2}{\hbar} a_{t-T}^{\prime}\right) \beta_{h / 2}\left(s^{\prime}\right)
\end{aligned}
$$

with $\beta_{\hbar}$ the Bernoulli measure, i.e. $\beta_{\hbar}=\frac{1}{2}\left(\delta_{\hbar}+\delta_{-h}\right)$. Then $\Xi$ satisfies the following equation:

$$
\begin{aligned}
& \frac{\partial \Xi}{\partial t}(t, q, p, s)+\int_{\mathbb{R}^{2 n+1} \backslash\{0\}} d v_{t q p s}\left(q^{\prime}, p^{\prime}, s^{\prime}\right) \\
& \quad \times\left\{\Xi\left(t, q+q^{\prime}, p+p^{\prime}, s+s^{\prime}\right)-\Xi(t, q, p, s)\right\}=0
\end{aligned}
$$

with the boundary condition $\lim _{t \uparrow T} \Xi(t, q, p, s)=e^{(i / h) s} W_{\hbar}(q, p)$.

The next theorem is an application of a known result of the theory of stochastic differential Eqs. [17] III. p. 176 
Theorem 2.4. There exists a locally infinitely divisible process with values in $\mathbb{R}^{2 n+1}$,

$$
t<T \rightarrow\left(Q_{t}^{I}(T), \quad P_{t}^{I}(T), \quad S_{t}^{I}(T)\right)
$$

such that $\left(Q_{T}^{I}(T), P_{T}^{I}(T), S_{T}^{I}(T)\right)=(q, p, s)$ a.s. whose generator has a modification $A_{t}$ satisfying

$$
\left(A_{t} f\right)(q, p, s)=\int_{\mathbb{R}^{2 n+1} \backslash\{0\}} d v_{\text {tqps }}\left(q^{\prime}, p^{\prime}, s^{\prime}\right)\left(f\left(q+q^{\prime}, p+p^{\prime}, s+s^{\prime}\right)-f(q, p, s)\right),
$$

such that $\Xi(t, q, p, s)=\mathbb{E}\left[\Xi\left(t=T, Q_{t}^{I}(T), P_{t}^{I}(T), S_{t}^{I}(T)\right)\right]$ for all $f \in C_{b}^{1}\left(\mathbb{R}^{2 n+1}\right)$, where $C_{b}^{1}\left(\mathbb{R}^{2 n+1}\right)$ denotes the space of bounded continuous functions with bounded continuous derivative.

As a consequence one has the following

Corollary 2.5. For every $T \geqq 0$,

$$
W_{h T}^{I}(q, p)=\exp \left\{4 \frac{T|\mu|}{\hbar}\right\} \mathbb{E}\left[\exp \left\{\frac{i}{h} S^{I}(T)\right\} W_{h}\left(Q^{I}(T), P^{I}(T)\right)\right] .
$$

For the sake of completeness we derive a similar result for the time evolution of the Wigner functions, expressing the Wigner function at time $t$ as an expectation over a Markov process of its initial value. Such a representation has already been derived in [1] (see also [18] for a similar representation), but with a slightly different stochastic process.

Theorem 2.6. There exists an infinitely locally divisible Markov process $(Q(T), P(T)$, $S(T)) \in \mathbb{R}^{2 n+1}$, whose generator A has a modification which is given for all $f \in C_{b}^{1}\left(\mathbb{R}^{2 n+1}\right)$ by

$$
\begin{aligned}
(A f)(q, p, s)= & \int_{\mathbb{R}^{2 n+1} \backslash\{0\}} d v_{T q p s}\left(q^{\prime}, p^{\prime}, s^{\prime}\right)\left\{f\left(q+q^{\prime}, p+p^{\prime}, s+s^{\prime}\right)-f(q, p, s)\right\} \\
& +\sum_{i=1}^{n}\left\{\frac{p_{i}}{m_{i}} \frac{\partial}{\partial q_{i}} f(q, p)-m_{i} \omega_{i}^{2} q_{i} \frac{\partial}{\partial p_{i}} f(q, p)\right\} .
\end{aligned}
$$

For any Wigner function belonging to $C_{b}^{1}\left(\mathbb{R}^{2 n}\right)$ and for $T>0$ we have

$$
W_{h T}(q, p)=\exp \left(4 T \frac{|\mu|}{\hbar}\right) \mathbb{E}\left[\exp \left(\frac{i}{h} S(T)\right) W_{h}(Q(T), P(T))\right] .
$$

As already mentioned in [1], from these theorems we define for $t \geqq 0$ and for all $f \in C_{b}^{1}\left(\mathbb{R}^{2 n+1}\right)$,

$$
\left(\Phi_{h t} f\right)(q, p, s)=\mathbb{E}[f(Q(t), P(t), S(t))],
$$

$t \rightarrow \Phi_{h t}$ is a continuous flow on $C_{b}^{1}\left(\mathbb{R}^{2 n+1}\right)$. In a similar way one defines the family of mappings $(t \geqq 0)$ of $C_{b}^{1}\left(\mathbb{R}^{2 n+1}\right)$

$$
\left(\Phi_{h t}^{I} f\right)(q, p, s)=\mathbb{E}\left[f\left(Q^{I}(t), P^{I}(t), S^{I}(t)\right] .\right.
$$

Our main interest is in the flow $\Phi_{h t}$. However for technical reasons connected with the fact that the drift of the process $(Q(t), P(t), S(t))$ is not bounded, we shall first 
consider $\Phi_{h t}^{I}$, and we will show that the corresponding result for $\Phi_{h t}$ follows immediately.

\section{Classical Limits}

It has been noted in [19] that the equation governing the time evolution of the Wigner function (Eq. (3.34) in [2]) approaches, in the limit $\hbar \rightarrow 0$ the classical Liouville equation. Therefore, one can expect that the solution of the limit equation is close to the solution of the equation describing the time evolution of Wigner functions.

This can be done by gathering the previous results with those of Ventsel [6], estimating the probability for a path of the stochastic process $(Q(T), P(T), S(T)$ to be close from the classical trajectory.

Following [6] we consider the exponential moment $G_{h}^{I}(t, q, p, s, z), z=\left(z_{1}, z_{2}, z_{3}\right)$, $z_{i} \in \mathbb{R}^{n}, i=1,2, z_{3} \in \mathbb{R}$ :

$$
\begin{aligned}
G_{h}^{I}(t, q, p, s, z)= & \frac{2}{\hbar} \int d|\mu|\left(a^{\prime}\right)\left(1-\sin \left(\sigma\left(a_{T-t}^{\prime}, a\right)+\varphi\left(a^{\prime}\right)\right)\right) \\
& \cdot \exp \left\{\frac{\hbar}{2} q_{T-t}^{\prime} z_{1}+\frac{\hbar}{2} p_{T-t}^{\prime} z_{2}\right\} \cosh \left(\hbar z_{3}\right)+\frac{2}{\hbar} \int d|\mu|\left(a^{\prime}\right) \\
& \cdot \exp \left\{\frac{\hbar}{2} q_{T-t}^{\prime} z_{1}+\frac{\hbar}{2} p_{T-t}^{\prime} z_{2}\right\} \cosh \left(\frac{\hbar}{2} z_{3}\right)-4 \frac{|\mu|}{\hbar},
\end{aligned}
$$

and we assume that $G_{h}^{I}$ exists at least in a neighbourhood of the origin, which is equivalent to assume that $V$ can be analytically continued in a neighbourhood of the real space.

\section{Lemma 3.1.}

$$
z \in \mathbb{R}^{2 n+1} \rightarrow G_{h}^{I}(t, q, p, s, z)
$$

is a bounded convex continuous function. Furthermore,

$$
G_{\hbar}^{I}(t, q, p, s, 0)=0 .
$$

This lemma is obvious, except that for the last statement one uses the fact that $|\mu|$ is symmetric, which is a consequence of the reality of the potential.

The next lemma is also obvious:

Lemma 3.2. The Legendre transform $G_{\hbar}^{I *}$ of $G_{\hbar}^{I}$,

$$
G_{\hbar}^{I *}(t, q, p, s, \xi)=\sup _{z \in \mathbb{R}^{2 n+1}}\left\{z_{1} \xi_{1}+z_{2} \xi_{2}+z_{3} \xi_{3}-G_{h}^{I}(t, q, p, s, z)\right\}
$$

is a convex bounded (continuous) function. It reaches its minimum 0 for

$$
\xi_{1}=-\frac{\partial V}{\partial p}\left(q_{t}, p_{t}\right), \quad \xi_{2}=\frac{\partial V}{\partial q}\left(q_{t}, p_{t}\right), \quad \xi_{3}=0
$$

independently of $\hbar$. 
Consequently, one can define an action function

$$
I_{t}^{\hbar}(\varphi)=\int_{0}^{t} d t G_{\hbar}^{I *}\left(t, q_{t}, p_{t}, s_{t}, \dot{q}_{t}, \dot{p}_{t}, \dot{s}_{t}\right), \varphi_{t}=\left(q_{t}, p_{t}, s_{t}\right)
$$

whose minimum defines the classical path.

We are interested in the behaviour for small $\hbar$ of the process $\left(Q^{I}(T), P^{I}(T), S^{I}(T)\right)$. We remark first that

$$
G_{1}^{I}(t, q, p, s, z)=\hbar G_{\hbar}^{I}\left(t, q, p, s, \frac{1}{\hbar} z\right)
$$

is independent of $\hbar$.

The next result is easy as well:

Lemma 3.3. Let

$$
G_{0}^{I}(z)=\sup _{q, p, s, t} G_{1}^{I}(t, q, p, s, z) .
$$

It is a convex continuous function bounded on every compact. Furthermore, we obviously have

$$
G_{1}^{I}(t, q, p, s, z) \leqq G_{0}^{I}(z), \quad G_{0}^{I}(0)=0 .
$$

As defined, $G_{0}^{I}(z)$ has all the properties required to apply Ventsel's results except the fact that it is possibly infinite. However, if

$$
\begin{aligned}
G_{1}^{I}(t, z)= & \int d|\mu|\left(a^{\prime}\right) \exp \left\{\frac{1}{2} q_{T-t}^{\prime} z_{1}+\frac{1}{2} p_{T-t}^{\prime} z_{2}\right\} \\
& \left(4 \cosh \left(\hbar z_{3}\right)+2 \cosh \left(\frac{\hbar}{2} z_{3}\right)\right)-4|\mu| .
\end{aligned}
$$

Then $G_{1}^{I}(t, q, p, s, z) \leqq G_{1}^{I}(t, z)$. Furthermore, $G_{1}^{I}(t, z) \leqq G_{1}^{I}(z)$, where

$$
G_{1}^{I}(z)=\int d|\mu|\left(a^{\prime}\right) \exp \left\{\frac{1}{2}\|(q, p)\| \cdot\left\|\left(z_{1}, z_{2}\right)\right\|\right\}\left(4 \cosh \left(z_{3}\right)+2 \cosh \left(\frac{1}{2} z_{3}\right)\right)-4|\mu|,
$$

where $\|(q, p)\|$ is the Euclidean norm on $\mathbb{R}^{2 n}$.

We are now in position to apply the main result of [6] (Theorem 2.1): If $\rho$ is the Sup norm distance between two trajectories viz:

$$
\rho_{O T}(\varphi, \psi)=\operatorname{Sup}_{t \in[0, T]} \sqrt{\sum_{i=1}^{2 n+1}\left(\varphi_{i}(t)-\psi_{i}(t)\right)^{2}}
$$

and if the tublet $\Phi_{q p s}(\varepsilon)$ around the classical path in $\mathbb{R}^{2 n+1}$ is defined by

$$
\Phi_{q p s}(\varepsilon)=\left\{\varphi ; \varphi(0)=(q, p, s) ; I_{T}^{h}(\varphi) \leqq \varepsilon\right\},
$$

then for any positive $\delta, \gamma, \varepsilon_{0}$, for sufficiently small $\hbar$ and for all $q, p, s, \in \mathbb{R}^{2 n+1}$ and $\varepsilon \leqq \varepsilon_{0}$

$$
\operatorname{Prob}\left\{\rho_{O T}\left(\left(Q_{\hbar}^{I}, P_{\hbar}^{I}, S_{\hbar}^{I}\right), \Phi_{q p s}(\varepsilon)\right) \geqq \delta\right\} \leqq \exp \left\{-\frac{1}{\hbar}(\varepsilon-\gamma)\right\} .
$$

From this estimate it follows that the quantum flow defined in (2.17) tends in the limit where $\hbar$ goes to zero toward the corresponding classical flow. More precisely, we have the 
Proposition 3.4. Let $\Phi_{h t}^{I}$ be the mapping for $t>0$ of $C_{b}^{1}\left(\mathbb{R}^{2 n+1}\right)$ defined by

$$
\begin{gathered}
\left(\Phi_{h t}^{I} F\right)(q, p, s)=\mathbb{E}\left[F\left(Q^{I}(t), P_{t}^{I}(t), S^{I}(t)\right)\right],(q, p, s) \in \mathbb{R}^{2 n+1}, \\
\lim _{t \downarrow 0}\left(\Phi_{h t}^{I} F\right)(q, p, s)=F\left(q_{t}^{I}, p_{t}^{I}, s_{t}^{I}\right),
\end{gathered}
$$

where the $q_{t}^{I}, p_{t}^{I}, s_{t}^{I}$ satisfy:

$$
\dot{q}_{t}^{I}=-\frac{\partial V}{\partial p}\left(q_{t}^{I}, p_{t}^{I}\right), \quad \dot{p}_{t}^{I}=\frac{\partial V}{\partial q}\left(q_{t}^{I}, p_{t}^{I}\right), \quad \dot{s}_{t}^{I}=0
$$

with the initial condition $\left(q_{t=0}^{I}, p_{t=0}^{I}, s_{t=0}^{I}\right)=(q, p, s)$.

Proof. For $\varepsilon>0$, let us introduce the characteristic function $\chi_{\varepsilon}$ of the set $\Phi_{q p s}(\varepsilon)$ and observe that:

$$
\begin{aligned}
A= & \left|\mathbb{E}\left[\Xi\left(Q_{t}^{I} \cdots\right)\right]-\Xi\left(q_{t}^{I} \cdots\right)\right| \leqq\left|\mathbb{E}\left[\left(\Xi\left(Q_{t}^{I} \cdots\right)-\Xi\left(q_{t}^{I} \cdots\right)\right) \chi_{\varepsilon}\right]\right| \\
& +\left|\mathbb{E}\left[\left(\Xi\left(Q_{t}^{I} \cdots\right)-\Xi\left(q_{t}^{I} \cdots\right)\right)\left(1-\chi_{\varepsilon}\right)\right]\right| .
\end{aligned}
$$

$\chi_{\varepsilon}$ is measurable as a consequence of [6]. We have then

$$
A \leqq \operatorname{Sup}_{\Phi_{q p s}(\varepsilon)}\left|\Xi\left(Q_{t}^{I} \cdots\right)-\Xi\left(q_{t}^{I} \cdots\right)\right|+2\|\Xi\| \mathbb{E}\left[1-\chi_{\varepsilon}\right],
$$

where $\|\Xi\|$ is the Sup norm of $\Xi$ viz:

$$
\|\Xi\|=\operatorname{Sup}_{q p s}|\Xi(q, p, s)|
$$

The continuity of $\Phi_{q p s}(\varepsilon)$ implies that one can choose $\varepsilon$ so small that the first term is small. Then choosing $\hbar$ small enough makes the second term arbitrary small.

As a corollary we give now our main result.

The quantum evolution approaches as $\hbar$ goes to zero the classical one viz.

Theorem 3.5. Let $\Phi_{h t}$ be the quantum flow defined on $C_{b}^{1}\left(\mathbb{R}^{2 n+1}\right)$ by

$$
\left(\Phi_{h t} F\right)(q, p, s)=\mathbb{E}\left(F\left(Q_{t}, P_{t}, S_{t}\right)\right) .
$$

Then

$$
\lim _{\hbar \downarrow 0}\left(\Phi_{h t} F\right)(q, p, s)=F\left(q_{t}, p_{t}, s\right),
$$

where $q_{t}, p_{t}$, are the solutions of the Hamilton equations

$$
\dot{q}=-\frac{\partial H}{\partial p}, \quad \dot{p}=\frac{\partial H}{\partial q},
$$

with initial condition $\left(q_{t}, p_{t}\right)_{t=0}=(q, p)$.

Proof. From the very definition of $\Phi_{h t}^{I}$ and $\Phi_{h t}$, one has that

$$
\Phi_{h t}=\Phi_{h t}^{I} \circ\left(\Phi_{t}^{0}\right)^{-1}
$$

But as it was remarked, $\Phi_{t}^{0}$ is independent of $\hbar$. The result follows from the following observation: 
Let $q_{t}^{I}, p_{t}^{I}$ be the solutions of the system of equations:

$$
\dot{q}_{t}^{I}=-\frac{\partial V}{\partial p} \quad \dot{p}_{t}^{I}=\frac{\partial V}{\partial q}
$$

with initial conditions $\left(q_{t}^{I}, p_{t}^{I}\right)_{t=0}=(q, p)$. Let

$$
\begin{aligned}
& Q_{t}(q, p)_{i}=\cos \left(\omega_{i} t\right)\left(q_{t}^{I}\right)_{i}-\frac{1}{m_{i} \omega_{i}} \sin \left(\omega_{i} t\right)\left(p_{t}^{I}\right)_{i}, \\
& P_{t}(q, p)_{i}=m_{i} \omega_{i} \sin \left(\omega_{i} t\right)\left(q_{t}^{I}\right)_{i}+\cos \left(\omega_{i} t\right)\left(p_{t}^{I}\right)_{i} .
\end{aligned}
$$

Then $Q_{t}$ and $P_{t}$ satisfy

$$
\dot{Q}_{t}=-\frac{\partial H}{\partial q}, \quad \dot{P}_{t}=\frac{\partial H}{\partial p}
$$

with the same initial conditions as $q_{t}^{I}, p_{t}^{I}$, and

$$
H(q, p)=\sum_{i=1}^{n}\left\{\frac{p_{i}^{2}}{2 m_{i}}+\frac{m_{i} \omega_{i}^{2}}{2} q_{i}^{2}\right\}+V(q, p) .
$$

Then the result of the theorem is obvious.

\section{Classical Limit of Wigner Functions}

Previous results cannot be directly applied to the study of the classical limit $(\hbar \downarrow 0)$ of Wigner functions. Indeed, from the representation derived in Sect. 2 the function under the expectation contains $\hbar$ explicitly, i.e.

$$
\left(\Phi_{h t} f\right)(q, p)=\exp \left\{4 \frac{t}{\hbar}|\mu|\right\} \mathbb{E}\left[\exp \left\{\frac{i}{h} S(t)\right\} f(Q(t), P(t))\right],
$$

where $(Q(t), P(t), S(t))$ is the stochastic process defined in Theorem 2.6.

However, from the observation that

$$
\exp \left\{4 \frac{t}{h}|\mu|\right\} \mathbb{E}\left[\exp \left\{\frac{i}{h} S(t)\right\}\right]=1,
$$

one can expect that the integral has an oscillatory character which implies that the limit exists and is precisely the one given by the classical motion, at least for sufficiently smooth initial conditions. More precisely, one has the following (see also [20] for connected results)

Theorem 4.1. Let $t \rightarrow\left(q_{t}, p_{t}\right)$ be the solution of the classical equation of motion with initial condition $(q, p) \in \mathbb{R}^{2 n}$. Assume that $f_{0}: \mathbb{R}^{2 n} \rightarrow \mathbb{C}$ belongs to $C_{b}^{1}\left(\mathbb{R}^{2 n}\right)$ and is such that $t \rightarrow f_{0}\left(q_{t}, p_{t}\right)$ is analytic (for $\left.t<T\right)$, then

$$
\lim _{h \rightarrow 0_{+}} \exp \left\{4 \frac{t|\mu|}{\hbar}\right\} \mathbb{E}\left[\exp \left\{\frac{i}{h} S(t)\right\} f_{0}(Q(t), P(t))\right]=f_{0}\left(q_{t}, p_{t}\right)
$$

for $t<T$.

Proof. Observe that it is sufficient to prove the result for the interaction picture (see 
remark Sect. 2). For this one has the explicit representation

$$
\begin{aligned}
\exp \left\{4 \frac{t}{\hbar}|\mu|\right\} \mathbb{E}\left[\exp \left\{\frac{i}{h} S(t)\right\} f_{0}\left(Q^{I}(t), P^{I}(t)\right)\right] \\
=\sum_{n \geqq 0} \int_{0}^{t} d t_{n} \int_{0}^{t_{n}} d t_{n-1} \cdots \int_{0}^{t_{2}} d t_{1}\left(\frac{2}{\hbar}\right)^{n} \int d|\mu|\left(a_{n}^{\prime}\right) \int \cdots \int d|\mu|\left(a_{1}^{\prime}\right) \\
\cdot g\left(t_{n}, a, a_{n}^{\prime}\right) g\left(t_{n-1}, a+\frac{\hbar}{2} a_{n}^{\prime}, a_{n-1}^{\prime}\right) \\
\quad \cdots g\left(t_{1}, a+\frac{\hbar}{2}\left(a_{n}^{\prime}+\cdots+a_{2}^{\prime}\right), a_{1}^{\prime}\right) f_{0}\left(a+\frac{\hbar}{2}\left(a_{n}^{\prime}+\cdots+a_{1}^{\prime}\right)\right),
\end{aligned}
$$

where

$$
g\left(t, a, a^{\prime}\right)=\sin \left(\sigma\left(a_{t}^{\prime}, a\right)+\varphi\left(a^{\prime}\right)\right) .
$$

We can rewrite the previous expression as an integral over the instants of jump of the process

$$
\exp \left\{4 \frac{t}{\hbar}|\mu|\right\} \mathbb{E}\left[\exp \left\{\frac{i}{h} S(t)\right\} f_{0}\left(Q^{I}(t), P^{I}(t)\right)\right]=\int_{\Omega} P(d \omega) F\left(\hbar, a, f_{0}\right)(\omega) .
$$

$\omega \in \Omega$ means that $\omega \equiv\left(n, 0<t_{1}<\cdots<t_{n}<t\right)$ and

$$
\begin{aligned}
\int P(d \omega) F(\omega)= & \sum_{n \geq 0} \int_{0}^{t} d t_{n} \int_{0}^{t_{n}} d t_{n-1} \cdots \int_{0}^{t_{2}} d t_{1} F\left(t_{1}, \ldots, t_{n}\right) \\
F\left(\hbar, a, f_{0}\right)(\omega)= & \left(\frac{2}{\hbar}\right)^{n} \int \cdots \int \prod_{i=1}^{n} d|\mu|\left(a_{i}^{\prime}\right) g\left(t_{n}, a, a_{n}^{\prime}\right) \\
& \cdot g\left(t_{n-1}, a+\frac{\hbar}{2} a_{n}^{\prime}, a_{n-1}^{\prime}\right) \cdots f_{0}\left(a+\frac{\hbar}{2}\left(a_{n}^{\prime}+\cdots+a_{1}^{\prime}\right)\right) .
\end{aligned}
$$

There exists an integrable majorant $F_{0}$ for $F\left(\hbar, a, f_{0}\right)$,

$$
F_{0}(\omega)=\frac{1}{n !} \operatorname{Sup}_{a}\left|\frac{d^{n}}{d \hbar^{n}}\left(\hbar^{n} F\left(\hbar, a, f_{0}\right)(\omega)\right) \uparrow_{\hbar=0}\right| \text {. }
$$

$F_{0}$ is a majorant since the measure $|\mu|$ is symmetric and $g$ is antisymmetric in the variable $a^{\prime}$. This implies immediately that $\left(d^{k} / d \hbar^{k}\right)\left(\hbar^{n} F\left(\hbar, a, f_{0}\right)(\omega)\right) \uparrow_{\hbar=0}=0$ for $k \leqq n-1$. On the other hand, $F_{0}$ is integrable since the perturbation series for the solution of the classical equation of motion

$$
\frac{d f}{d t}\left(q_{t}, p_{t}\right)=\left\{H\left(q_{t}, p_{t}\right), f\left(q_{t}, p_{t}\right)\right\}
$$

where $\{$,$\} denotes the Poisson brackets, was assumed to be absolutely convergent.$

To conclude the proof one has to observe that pointwise in $\omega$

$$
\lim _{h \rightarrow 0} F\left(\hbar, a, f_{0}\right)(\omega)=\left\{V\left(a_{t_{n}}\right),\left\{V\left(a_{t_{n-1}}\right) \cdots\left\{V\left(a_{t_{1}}\right), f_{0}(a)\right\} \cdots\right\}\right\}
$$


For the sake of completeness let us mention the connections of our results to those obtained by K. Hepp [14]. Actually, the Wigner functions contain explicitly $\hbar$ viz

$$
W_{h}(a)=(\hbar \pi)^{-n} \operatorname{tr}\left(R W_{2 a / \hbar}^{*} \Pi\right) .
$$

For coherent states (see e.g. [21]) one has explicitly:

$$
W_{h q_{0} p_{0}}(q, p)=\frac{1}{(2 \pi \hbar)^{n}} \exp \left\{\frac{1}{2 \hbar}\left[\left(q-q_{0}\right)^{2}+\left(p-p_{0}\right)\right]^{2}\right\} .
$$

Namely, they are smooth approximations of Dirac measures on phase space $\mathbb{R}^{2 n}$. Consequently, one has:

Proposition 4.2. Under the same assumptions as in Theorem 4.1

$$
\lim _{h \downarrow 0}\left\langle q_{0} p_{0}\left|\exp \left\{\frac{i t}{\hbar} H\right\} Q\left(f_{0}\right) \exp \left\{-\frac{i t}{\hbar} H\right\}\right| q_{0} p_{0}\right\rangle=f_{0}\left(q_{t}, p_{t}\right),
$$

where $\left|q_{0} p_{0}\right\rangle$ is the coherent state defined in (4.8), $Q\left(f_{0}\right)$ the quantum operator associated to the function $f_{0}$, and $\left(q_{t}, p_{t}\right)$ the solution of the classical equation of motion with initial condition $\left(q_{0}, p_{0}\right) \in \mathbb{R}^{2 n}$.

Related results about the classical limit of Wigner functions can be obtained for one dimensional systems and more generally for completely integrable systems [22].

Acknowledgements It is a great pleasure to express our gratitude to Prof G Jona-Lasinio for introducing us to the theory of large deviations as well as for useful comments We also thank Prof A Badrikian for discussions

One of us (MS) is very grateful to Prof $\mathrm{L}$ Streit for his kind invitation at the Zentrum für interdisziplinäre Forschung as well as for the stimulating atmosphere he created there

\section{References}

1 Albeverio, S, Blanchard, $\mathrm{Ph}$, Combe, $\mathrm{Ph}, \mathrm{H} \phi$ egh-Krohn, $\mathrm{R}$, Sirugue, M : Local relativistic invariant flow for quantum fields Commun Math Phys 90, 329-351 (1983)

2 Combe, Ph, Guerra, F, Rodriguez, R, Sirugue, M, Sirugue-Collin, M : Quantum dynamical time evolutions as stochastic flows in phase space Proceeding of the VIIth conference of I A M P, Boulder-Colorado (August 1983) Physica 124A, 561-574 (1984)

3. Donsker, M D, Varadhán, S R S : Asymptotic evaluation of certain markov expectations for large time Commun Pure Appl Math I, 28, 1-47 (1975) II, 29, 279-301 (1976); III, 29, 389-461 (1976)

4 Ventsel', A. D : Rough limit theorem on large deviations for Markov stochastic processes I Theory Probab Appl 21, 227-242 (1976)

5 Ventsel', A D : Rough limit theorem on large deviations for Markov stochastic processes II Theory Probab Appl 21, 499-512 (1976)

6. Ventsel', A D : Rough limit theorem on large deviations for Markov stochastic processes III Theory Probab Appl 24, 675-692 (1979)

7 Azencott, R : Grandes déviations et applications Cours de probabilité de Saint-Flour Lecture Notes in Mathematics, Vol 774, Berlin, Heidelberg, New York: Springer 1978

8 Jona-Lasinio, G, Martinelli, F, Scoppola, E : New approach to the semi-classical limit of quantum mechanics I Multiple tunnelings in one dimension Commun Math Phys 80, 223-254 (1981)

9 Jona-Lasinio, G., Martinelli, F, Scoppola E : The semi-classical limit of quantum mechanics: a qualitative theory via stochastic mechanics Phys Rep 77, 313-327 (1981) 
10. Azencott, R Doss, H : L'équation de Schrödinger quand $\hbar \rightarrow 0$ : une approche probabiliste Stochastic aspects of classical and quantum systems proceedings Marseille In: Lecture Notes in Mathematics, vol 1109, pp 1-17, Berlin, Heldelberg, New York: Springer 1985

11 Simon, B.: Instantons, double wells and large deviations Bull A M S March 1983

12 De Angelis, G F., Jona-Lasinio, G, Sirugue, M : Probabilistic solutions of Pauli type equations J Phys A 16, 2433-2444 (1983)

13 Gihman, I I, Skorohod, A. V : Stochastic differential equations Berlin, Heidelberg, New York: Springer 1972

14 Hepp, K.: The classical limit for quantum mechanical correlation functions Commun Math Phys 35, 265-277 (1974)

15 Fano, U : Description of states in quantum mechanics by density matrix and operator techniques Rev. Mod Phys. 29, 74-93 (1957)

16. Weyl, H.: Gruppentheorie und Quantenmechanik, Leipzig: Hirzel, 1931

17. Gihman, I. I, Skohorod, A V.: The theory of stochastic processes I, II and III Berlin, Heidelberg, New York: Springer 1974

18. Bertrand, J., Gaveau, G.: Transformations canoniques et renormalisation pour certaines équations d'évolution. J. Funct Anal. 50, 81-99 (1983)

19. Moyal, J. E.: Quantum mechanics as a statistical theory Proc Camb Phil Soc 45, 99-124 (1949)

20. Bertrand, J., Rideau, G : Stochastic jump processes in the phase space representation of quantum mechanics. Proceedings of the VIth conference of I A M P, Berlin (1981) Lecture Notes in Physics, Vol 153, Berlin, Heidelberg, New York: Springer 1982; Stochastic processes and evolution of quantum observables Preprint Paris VII, March 1983

21 Grossmann, A, Seiler, R.: Heat equation on phase space and the classical limit of quantum mechanical expectation values Commun Math. Phys 48, 195-197 (1976)

22 Sirugue, M, Sirugue-Collin, M, Truman A : Semi-classical approximation and microcanonical ensemble Ann Inst. Henri Poincaré 41, 429-444 (1984)

Communicated by J. Lascoux

Received January, 1984 
